\title{
Effectiveness, safety and acceptability of no-test medical abortion provided via telemedicine: a national cohort study
}

\author{
Abigail Aiken ${ }^{1}$, Patricia Lohr ${ }^{2}$, Jonathan Lord ${ }^{3}$, Nabanita Ghosh $^{4}$, and Jennifer Starling ${ }^{5}$ \\ ${ }^{1}$ University of Texas at Austin \\ ${ }^{2}$ British Pregnancy Advisory Service \\ ${ }^{3}$ MSI Reproductive Choices \\ ${ }^{4}$ National Unplanned Pregnancy Advisory Service \\ ${ }^{5}$ Mathematica Policy Research inc Cambridge Office
}

December 11, 2020

\begin{abstract}
Objective To compare the effectiveness, safety and acceptability of medical abortion before and after the introduction of notest telemedicine Design Cohort study Setting The three main abortion providers in England Population All patients having an early medical abortion (comprising $85 \%$ of all medical abortions performed nationally) Methods Comparison of no-test telemedicine hybrid model vs. traditional model (blanket in-person provision including ultrasound), adjusted for baseline differences Main outcome measures Access: waiting time, gestation Effectiveness: successful medical abortion Safety: significant adverse events; ectopic pregnancy and late gestation Acceptability: Patient-reported outcomes Results 52,142 medical abortions were conducted, 29,984 in the telemedicine-hybrid cohort and 22,158 in the traditional cohort. Mean waiting times were 4.2 days shorter in the telemedicine-hybrid cohort and $40 \%$ were [?] 6 weeks' gestation vs. $25 \%$ in the traditional cohort (p<0.001). There was no difference in success rates $(98.8 \%$ vs. $98.2 \%, \mathrm{p}=1.0)$, nor in prevalence of serious adverse events $(0.02 \%$ vs. $0.04 \%$, $\mathrm{p}=0.557)$. Incidence of ectopic pregnancy was equivalent in both cohorts $(0.2 \%, \mathrm{p}=0.796) ; 0.04 \%$ of abortions appeared to have been provided after 10 weeks' gestation with all completed safely at home. In the telemedicine-hybrid cohort, effectiveness was higher in the telemedicine group vs. the in-person group (99.2\% vs. 98.1\%, p<0.001). Acceptability was high $(96 \%$ satisfied), $80 \%$ reported a future preference for telemedicine, and none reported that they were unable to consult in private using teleconsultation. Conclusions Medical abortion provided through a hybrid model that includes no-test telemedicine without ultrasound is effective, safe, acceptable, and improves access to care. Funding None
\end{abstract}

Effectiveness, safety and acceptability of no-test medical abortion provided via telemedicine: a national cohort study

Authors:

Abigail Aiken, MD, MPH, PhD

Associate Professor of Public Affairs, LBJ School of Public Affairs

University of Texas at Austin

araa2@utexas.edu

Orcid: 0000-0002-1555-3025

Patricia A Lohr, MD MPH

Medical Director, British Pregnancy Advisory Service 
Stratford upon Avon, CV37 9BF

patricia.lohr@bpas.org

Orcid: 0000-0003-1862-5730

Jonathan Lord*, MD FRCOG

Medical Director, MSI Reproductive Choices. Consultant gynaecologist.

1 Conway Street, Fitzroy Square, London W1T 6LP

J.lord@exeter.ac.uk

Orcid: 0000-0003-2819-5973

Nabanita Ghosh, FRCOG

Medical Director, National Unplanned Pregnancy Advisory Service (NUPAS)

nghosh@nhs.net

Jennifer Starling, PhD

Statistician, Mathematica Policy Research, Cambridge, MA, 02139

jstarling@mathematica-mpr.com

Orcid: 0000-0003-4765-360X

$*=$ Corresponding author

MeSH / Keywords:

Abortion, Induced [E04.520.050]

Telemedicine [N04.590.374.800]

Ambulatory Care Facilities [N02.278.035]

Health Planning [N03.349]

Pregnancy Complications [C13.703]

Mifepristone [D04.210.500.365.415.580]

Misoprostol [D23.469.700.660.500]

Word count: 3485 (abstract 247)

\section{Abstract}

Objective

To compare the effectiveness, safety and acceptability of medical abortion before and after the introduction of no-test telemedicine

Design

Cohort study

\section{Setting}

The three main abortion providers in England

\section{Population}

All patients having an early medical abortion (comprising $85 \%$ of all medical abortions performed nationally) 


\section{Methods}

Comparison of no-test telemedicine hybrid model vs. traditional model (blanket in-person provision including ultrasound), adjusted for baseline differences

Main outcome measures

Access : waiting time, gestation

Effectiveness : successful medical abortion

Safety : significant adverse events; ectopic pregnancy and late gestation

Acceptability : Patient-reported outcomes

\section{Results}

52,142 medical abortions were conducted, 29,984 in the telemedicine-hybrid cohort and 22,158 in the traditional cohort. Mean waiting times were 4.2 days shorter in the telemedicine-hybrid cohort and $40 \%$ were [?] 6 weeks' gestation vs. $25 \%$ in the traditional cohort $(\mathrm{p}<0.001)$. There was no difference in success rates ( $98.8 \%$ vs. $98.2 \%, \mathrm{p}=1.0)$, nor in prevalence of serious adverse events $(0.02 \%$ vs. $0.04 \%, \mathrm{p}=0.557)$. Incidence of ectopic pregnancy was equivalent in both cohorts $(0.2 \%, \mathrm{p}=0.796) ; 0.04 \%$ of abortions appeared to have been provided after 10 weeks' gestation with all completed safely at home. In the telemedicine-hybrid cohort, effectiveness was higher in the telemedicine group vs. the in-person group (99.2\% vs. $98.1 \%, \mathrm{p}<0.001)$. Acceptability was high (96\% satisfied), $80 \%$ reported a future preference for telemedicine, and none reported that they were unable to consult in private using teleconsultation.

\section{Conclusions}

Medical abortion provided through a hybrid model that includes no-test telemedicine without ultrasound is effective, safe, acceptable, and improves access to care.

\section{Funding}

None

\section{Tweetable Abstract}

Compelling evidence from 52,145 women shows telemedicine abortion is safe, effective \& improves care

\section{Introduction}

Improved access to abortion care would deliver significant advantages for both healthcare systems and the women who use them. Abortion is a common reason for needing healthcare - the global abortion rate is estimated at 39 abortions per 1000 women aged 15-49 years,(1) with $28 \%$ of all pregnancies in developed countries ending in abortion.(2) There is clear evidence that restricting access to abortion does not reduce abortion rates, it simply makes abortion less safe. $(1,3)$ Improving access is likely to benefit those who are most vulnerable,(4) especially in resource-poor settings or where care has to be self-funded. In its 2019 guideline on abortion care, the National Institute for Health and Care Excellence (NICE) stated that improving access to abortion was a key priority.(5)

Telemedicine, the use of information and communication technologies to improve patient outcomes by increasing access to care and medical information,(6) has been noted to decrease costs and increase convenience and safety.(7) It is an established service delivery model for abortion care in many settings,(8) and it is recommended to improve access.(4) The COVID-19 pandemic required urgent action by clinicians and policymakers to ensure delivery of essential health services, including abortion. In March 2020, the Royal College of Obstetricians and Gynaecologists (RCOG) published guidelines to safeguard abortion care in the UK.(9) The guidelines profoundly changed the way medical abortion care is delivered in Great Britain. Prior to the emergence of COVID-19, all patients seeking medical abortion were required to attend in-person to receive an ultrasound scan and have mifepristone administered within the clinic. Under the new guidelines, 
consultations were encouraged to take place by telephone or video call; an ultrasound scan was required only if indicated. All the British governments had issued emergency legal orders by March $30^{\text {th }} 2020$ to allow mifepristone to be used at home along with misoprostol up to 10 weeks' gestation.(10-12) These approvals permitted abortion providers to implement a telemedical service delivery model for medical abortion.

Great Britain's new pathway for no-test telemedicine abortion is unusual among existing models because it is fully remote: no clinic visit, tests or ultrasound scan are required and both mifepristone and misoprostol are delivered by mail or collected from a clinic for use at home. This new service model thus presents an important opportunity to evaluate a potentially better way to provide medical abortion that could improve access and reduce the barriers posed by in-person care.(13) We examined and compared the effectiveness, safety and acceptability of medical abortion provided up to 10 weeks' gestation before and after the service model change.

\section{Methods}

\section{Population and Cohorts}

The study population comprised all patients who accessed an early medical abortion (EMA) at the three largest abortion providers in England and Wales - British Pregnancy Advisory Service (BPAS), MSI Reproductive Choices (MSUK), and the National Unplanned Pregnancy Advisory Service (NUPAS) - two months before and after the service model change. Medical abortion is defined as the use of medications to cause termination of a pregnancy without primary surgical intervention and 'EMA' applies to these procedures in the first trimester.(14) The recommended EMA regimen uses the anti-progestogen mifepristone in an oral dose of $200 \mathrm{mg}$ then, after $24-48$ hours, $800 \mathrm{mcg}$ of the prostaglandin analogue misoprostol by the sublingual, vaginal or buccal route.

Our dataset consisted of information on EMAs extracted directly from electronic service records and included fully de-identified patient clinical and demographic characteristics, as well as clinical outcomes. All data were extracted six weeks after the end of the study period to ensure the reporting of complications was as complete as possible.

Two cohorts were defined. The "traditional" cohort comprises all patients having an EMA between January $1^{\text {st }}$ and March 1st 2020, prior to service model change. All patients in this cohort received care using the traditional pathway that included an in-person assessment and an ultrasound scan. The "telemedicinehybrid" cohort comprises all patients accessing an EMA between April $6^{\text {th }}$ and June $30^{\text {th }} 2020$, in a two month period after the service model change at each provider. Patients in this cohort received care using no-test telemedicine if they had low risk of ectopic pregnancy and their self-reported last menstrual period (LMP) indicated a gestation of less than 10 weeks. Those who were not eligible for telemedicine had an inperson assessment with ultrasound. Providers followed organisation-specific evidence-based policies informed by the RCOG(9) and associated decision aid(15) (figures 1 and 2) and earlier RCOG and NICE guidelines for general abortion care including in-person medical abortion. $(16,17)$

In the traditional cohort, whilst some aspects of the pre-abortion consultation may have been carried out by phone, an in-person assessment with ultrasound scan and in-clinic administration of mifepristone were always required. In the telemedicine-hybrid cohort, we defined patients served by telemedicine as those who received medications for home use (either by mail or collection with minimal contact from a clinic) following a phone or video consultation and who did not receive an ultrasound scan or other tests.

We omitted the month of March 2020 because of the uncertainty and challenges posed by the emergence of COVID prior to the definitive service model change. The dates of the telemedicine-hybrid cohort take into account the roll-out of the telemedicine service across individual clinics within a provider network. For the analysis of ectopic pregnancies, we applied the timeframes stated above to all abortion consultations rather than all abortions provided because some patients with suspected ectopic pregnancies were referred to Early Pregnancy Assessment Units (EPAU) and did not proceed to an abortion.

\section{Outcome Measures}




\section{Access}

Waiting time and gestation at treatment were used to assess how the two models impacted access. Waiting time was defined as the interval from first contact with the abortion provider to when medication was dispensed (either in-person in clinic or posted). Gestation was recorded as that applicable on the date mifepristone was dispensed or provided. Analysis was of mean gestation and the proportion of abortions performed at [?]6 weeks' gestation.

\section{Effectiveness}

Effectiveness was defined as the proportion of medical abortions that were successful. Success was defined according to the MARE Guidelines as successful expulsion of an intrauterine pregnancy without need for surgical intervention.(14) Sub-categories of unsuccessful abortion included: surgical management of continuing pregnancy, surgical evacuation of retained products of conception, and continuing pregnancy where the patient opted for continuation or the outcome is unknown.

\section{Safety}

Safety was defined according to the proportions of medical abortions that involved one or more significant adverse events. We defined significant adverse events as haemorrhage requiring transfusion, significant infection requiring hospital admission, major surgery, and death. We also examined the incidence of ectopic pregnancy and when it was diagnosed in the care pathway. All patients referred for further diagnostics (e.g. serial $\beta$ hCG monitoring) but who had no further treatment are included in the ectopic pregnancy group although a proportion of these will be pregnancy of unknown locations (PULs) that includes failed early intrauterine pregnancies. We analysed the proportion of cases where treatment was reported to have occurred at [?]10 weeks' gestation in the telemedicine-hybrid cohort (this outcome does not apply to the traditional cohort since all gestations were determined by ultrasound scan).

\section{Acceptability}

Given the constraints of delivering healthcare during COVID-19 it was not possible to follow up with all patients to capture patient-reported outcomes. Two of the providers (BPAS and MSUK) collected data on patient feedback during the study period. Patients were invited to provide feedback one to three weeks after treatment, either by telephone using a structured interview tool (MSUK)(18) or using an online form (BPAS)(19). We analysed data on questions reporting on satisfaction, future preference and privacy. Although the questions were similar, only the MSUK survey included questions on the privacy of teleconsultation. All contact was by a non-clinician who had not been involved in the patient's care.

\section{Analysis}

The primary analysis was to assess whether the telemedicine-hybrid care model was non-inferior to the traditional service model. Non-inferiority is established by comparing effectiveness and safety in the two cohorts under the null hypothesis that the rates of successful abortion and serious adverse events are not different.

We first compared patient demographic and clinical characteristics between the cohorts to assess the need to covariate-adjust our hypothesis tests for systematic differences in the two groups that might affect abortion outcomes. All hypothesis tests were covariate adjusted for patient age, race/ethnicity, gestational age, parity, and prior abortions using logistic regression and weighted risk differences.(20)

We evaluated effectiveness by testing the alternative hypothesis that the rate of successful medical abortion in the telemedicine-hybrid cohort is lower than in the traditional cohort using a covariate-adjusted test of difference in proportions. We also performed a chi-squared test to evaluate whether the distribution of unsuccessful abortion sub-categories differed between the cohorts. We evaluated safety by testing the alternative hypothesis that significant adverse events occurred at higher rates in the telemedicine-hybrid cohort compared to the traditional cohort using a covariate-adjusted hypothesis test for difference of proportions. We also evaluated whether the prevalence of ectopic pregnancies managed before EMA and after EMA were 
different between the traditional and telemedicine-hybrid cohorts using chi-squared difference of proportions tests.

The secondary analysis was to compare effectiveness and safety of medical abortion for patients who received fully remote no-test telemedicine vs. in-person care in the telemedicine-hybrid cohort, primarily to assess whether any differences between the cohort service models were driven by one particular group. We evaluated patient demographic and clinical characteristic differences between the two groups to assess the need to covariate-adjust our hypothesis tests for systematic differences and all hypothesis tests were covariate adjusted for patient age, race/ethnicity, gestational age, parity, and prior abortions using the framework described above. We note, however, that these cohorts are fundamentally different despite covariate adjustment, as patients in the telemedicine-hybrid cohort were selected into in-person treatment based on characteristics that would affect the outcome of their abortion. We performed covariate-adjusted hypothesis tests under the null hypothesis of equal effectiveness and equal rates of significant adverse events in the telemedicine vs. in-person groups.

All analyses were performed using R, version 3.6.2. Statistical significance was defined using an alpha level of 0.05 .

\section{Ethics Approval}

The study was reviewed by the Institutional Review Board (IRB) at the University of Texas at Austin and a determination was made that the research did not meet the criteria for human subjects research as defined in the Common Rule (45 CFR 46) or FDA Regulations (21 CFR 56). Each provider ensured compliance with their own internal ethics and governance systems. The principles of the STROBE statement, and the MARE supplement, were followed.(14)

\section{Results}

In total, 52,142 medical abortions were provided during the study period; 22,158 in the traditional pathway and 29,984 in the telemedicine-hybrid cohort. Among those that took place in the telemedicine-hybrid cohort, $18,435(61 \%)$ were provided entirely via telemedicine and 11,549 (39\%) were provided in- person. Our sample represents $85 \%$ of the total number of medical abortions performed in England and Wales during the study period.(21) The clinical and demographic characteristics of patients in the two cohorts are described in Table 1.

\section{Access}

Mean waiting time to treatment declined from 10.7 days (SD 19.9) in the traditional pathway to 6.5 days (SD 13.5) in the telemedicine-hybrid cohort $(\mathrm{p}<0.001)$. Mean gestational age at treatment also declined in the telemedicine-hybrid cohort resulting in $40 \%$ of abortions performed at 6 weeks' gestation or less vs. $25 \%$ in the traditional pathway $(\mathrm{p}<0.001)$.

\section{Effectiveness}

Rates of successful medical abortion were high under both service delivery models (Table 2): 98.2\% in the traditional cohort vs. $98.8 \%$ in the telemedicine-hybrid cohort. We found no evidence of a lower success rate with the telemedicine-hybrid pathway $(\mathrm{p}=1.0)$. The distribution of the different sub-categories of unsuccessful medical abortion did not differ between the cohorts $(\mathrm{p}=0.268)$.

\section{Safety}

Significant adverse events in both cohorts were rare (Table 3). Haemorrhage requiring transfusion was reported in $8(0.04 \%)$ cases in the traditional cohort and $7(0.02 \%)$ cases in the telemedicine-hybrid cohort. No cases of significant infection requiring hospital admission, major surgery or death were reported. We found no evidence that significant adverse events were higher in the telemedicine-hybrid cohort $(\mathrm{p}=0.557)$.

The overall incidence of ectopic pregnancy was equivalent in both cohorts, $39(0.2 \%)$ in the traditional pathway and $49(0.2 \%)$ in the telemedicine pathway, $\mathrm{p}=0.796$ (Table 4 ). The proportions managed after 
EMA were not significantly different between the cohorts $(0.01 \%$ in the traditional pathway and $0.03 \%$ in the telemedicine-hybrid pathway, $\mathrm{p}=0.123)$. There were 11 cases $(0.04 \%)$ in the telemedicine-hybrid cohort where the gestational age after abortion was reported as being greater than the expected 10 weeks. In all these cases, the medical abortion was completed at home without additional medical complications.

\section{Acceptability}

Patient-reported outcome data was available from 2453 respondents. 96\% were "satisfied" or "very satisfied" with their care, or rated their experience as "good" or "very good". $80 \%$ reported that they would choose telemedicine in the future or that it was their preferred option, with $13 \%$ choosing in-person care and the remainder being unsure. No patient reported that they were unable to consult in private using teleconsultation $(\mathrm{n}=1243)$.

\section{Secondary Outcome Measures}

Comparison of clinical outcomes for the telemedicine vs. in-person groups in the telemedicine-hybrid cohort are shown in Tables S1-S3. Rates of successful medical abortion were higher in the telemedicine group (99.2\% vs. $98.1 \%, \mathrm{p}<0.001)$, and rates of significant adverse outcomes were not significantly different between the two groups, $3(0.02 \%)$ for telemedicine vs. $4(0.03 \%)$ for in-person, $\mathrm{p}=0.532$.

\section{Discussion}

\section{Main Findings}

We found that no-test telemedicine without routine ultrasound for medical abortion up to 10 weeks' gestation is an effective, safe and acceptable service model. Clinical outcomes with telemedicine are equivalent to in-person care and access to abortion care is better, with both waiting times and gestational age at the time of abortion significantly reduced. Further evidence that the new telemedicine-hybrid model improved access comes from a study showing that the rate of women seeking abortion medication outside the formal healthcare setting reduced significantly in the UK following implementation of telemedicine.(22) The most likely explanation is easier access as the opposite effect was seen in several other European countries who made no such provision. The implication is that those previously too vulnerable to attend in-person have been able to access care through telemedicine, potentially benefiting from the safeguarding, counselling and contraceptive services provided by regulated providers.

Our study confirms previous literature that medical abortion is safe and effective with low rates of significant complications.(23) The slight increase in effectiveness we observed in the group that received telemedicine - even after controlling for lower average gestational age compared to the in-person group - may be due to the ability of patients to better control the timing at which they took the medication.

The telemedicine-hybrid model resulted in very low rates of undiagnosed ectopic pregnancy and later than expected gestations. Although the rate of ectopic pregnancy in the general population in the UK and USA is reported as $1-2 \%,(24,25)$ the rate reported among patients having an abortion is 10 times lower, $(26)$ which is consistent with our findings. Ultrasound is not used to screen for ectopic pregnancy in the general population - it is only used where signs and symptoms suggest a need.(25) Routine screening of symptom-free women is associated with a high false positive rate when the prevalence of ectopic pregnancy is low, as is the case in women seeking abortion, and therefore it is unlikely there would be significant benefits.(27) There is no clinical justification for maintaining this inconsistency in care between women wishing to continue their pregnancies and those choosing EMA. $(28,29)$

However, given that over 200,000 people access abortion care each year in the UK alone, some will inevitably have an asymptomatic ectopic pregnancy and so will proceed with having mifepristone and misoprostol either through telemedicine or after a false negative scan. The essential issue for safety is that these are detected prior to causing harm rather than prior to beginning medical abortion treatment; treatment with mifepristone and misoprostol in itself will have no effect on an underlying ectopic pregnancy. Indeed, the reduction in waiting times afforded by the telemedicine model may facilitate earlier detection than traditional 
pathways where women present later, or are sent away to give additional time to visualise an intrauterine pregnancy on scan. Proceeding with early medical abortion without a scan may permit earlier diagnosis of a developing ectopic pregnancy owing to increased surveillance and index of suspicion, for example where there is minimal bleeding after misoprostol. $(9,16)$

The proportion of cases where gestational age was later than expected based on LMP was low, as might have been expected given the evidence that women can determine the gestational age of their pregnancy with reasonable accuracy by LMP alone.(29) Nevertheless inadvertent treatment of gestations over 10 weeks is inevitable and, consistent with our findings, the consequences for most are unlikely to be medically significant.(30) The 10 weeks' gestation limit in the English government's approval order is arbitrary, and not based on evidence of safety or effectiveness. The Scottish government did not stipulate a limit, leaving the decision to the discretion of the clinician in consultation with their patient. Moreover, the reported success of self-managed abortions at $>12-24$ weeks' gestation is $93 \%$, with safety similar to that expected in earlier gestations.(31)

\section{Strengths and Limitations}

While the study is not a clinical trial and we were not able to control the assignment of patients to treatment groups to conduct a direct comparison of fully remote telemedicine with in-person care, the policy changes precipitated by COVID-19 created a rare opportunity to conduct an evaluation of a major change in service delivery model with a very large sample size. We were able to evaluate the outcomes of both the telemedicinehybrid and traditional in-person services as they operate in the real world, and we were able to adjust for key covariates. A key strength of the study is the generalisability of our findings given that our sample included $85 \%$ of all medical abortions provided in England and Wales during the study period.

The main limitation of this study is that we were unable to actively follow-up patients post-abortion and therefore only significant adverse events can be reported with confidence. There is a potential gap in the consistency of reporting incidents, due to some complications not meeting the threshold of serious incidents, multiple routes of entry into the NHS and informal communication between the NHS and abortion providers. Although it is possible that some patients presented to other providers and a significant adverse event was not reported in our dataset, the risk management and reporting systems within the NHS are well defined, with serious incidents being routinely shared. Regulators expect providers to ensure actions are taken to mitigate risks to patients in the future. More importantly, there is no reason to suspect that any underreporting that did occur would be more likely in either cohort so as to introduce bias. The governing body of the NHS in England alerted all commissioners of the need to report incidents relating to telemedicine and there were review meetings of key stakeholders to ensure compliance. We consulted with regulators and national agencies to ensure that we accounted for reports made directly to them. The independent regulator of all health and social care services in England, the Care Quality Commission (CQC), confirmed that all cases reported directly to them through various routes, for example, statutory notifications and the central NHS database of patient safety incident reports (the National Reporting and Learning System (NRLS) and the Strategic Executive Information System (StEIS)), were known to the providers.

\section{Interpretation}

NICE conducted a systematic review and recommended using telemedicine to improve access to medical abortion care(4). Several models for using telemedicine to facilitate medical abortion have been described, but most existing trials are small, and many required attendances to have medicines administered or for an ultrasound scan or blood tests(8). Our study is the first to assess a real-world no-test telemedicine abortion care pathway in a national population. This new national model demonstrates how a permissive framework for medical abortion can deliver significant quality improvements to those needing to access abortion care.

\section{Conclusion}

This large study of 52,145 medical abortions demonstrates that incorporating no-test telemedicine into the care pathway is not inferior to the traditional pathway where all patients are seen in person and have an 
ultrasound scan. There are advantages - waiting times and gestation at abortion are reduced and it is highly rated by patients. There was no evidence of worse outcomes in failure rate, haemorrhage, need for surgery or in failure to detect ectopic pregnancy. In the $0.04 \%$ of cases where the abortion appeared to have been provided at over 10 weeks' gestation, these were all completed at home without additional medical complications. Given the advantages of improving access to care, especially in vulnerable groups and in resource-poor healthcare systems or where patients often have to fund their own care, the evidence is compelling that no-test telemedicine should become routine in the provision of abortion care.

\section{Acknowledgements}

We would like to thank the Care Quality Commission (CQC), NHS England and NHS Improvement (NHSE \& I), and the National Child Mortality Database (NCMD) for their advice and assistance in ensuring all complications reported within NHS systems have been included.

We are also enormously grateful to the many people who helped to ensure access to essential healthcare for women needing an abortion not only continued, but was improved during the COVID-19 pandemic. There are too many to list individually, but we would like to especially thank the following who not only helped to implement a complete service change within a few weeks, but also made this paper possible by ensuring the data was collected and accessible:

BPAS - Rebecca Blaylock, Steve Cheung, Pam Field, Stephen Franklin, Jeanette Taylor, Katherine Whitehouse

MSUK - Cat James, Andrew Lord, Kay Newey, Abigail Storan

NUPAS - Aaron Flaherty, Linda Leach, Leanne MacCaffrey

\section{Authors' contributions \& Declarations of Interest}

All authors have completed the ICMJE uniform disclosure form at www.icmje.org/coi_disclosure.pdf and declare: no support from any organisation for the submitted work; no financial relationships with any organisations that might have an interest in the submitted work in the previous three years; no other relationships or activities that could appear to have influenced the submitted work beyond that described under the roles held by each individual below.

Abigail Aiken - Initial concept, developing study protocol, data analysis, writing first draft and subsequent revisions, verification of data. Overall responsibility for data analysis \& principal investigator. Guarantor.

Member of the Council of the British Society of Abortion Care Providers (BSACP).

Nabanita Ghosh - Developing study protocol, revision of draft paper. Overall responsibility for conduct of study including data collection at NUPAS.

Nothing to disclose

Patricia A. Lohr - Initial concept, developing study protocol, writing first draft and subsequent revisions. Overall responsibility for conduct of study including data collection at BPAS.

Co-author / committee member of national guidelines cited in this paper from RCOG \& NICE; member RCOG abortion taskforce; council member of British Society of Abortion Care Providers (BSACP); Medical Director of BPAS.

Jonathan Lord - Initial concept, developing study protocol, writing first draft and subsequent revisions, co-ordination and liaison. Overall responsibility for conduct of study including data collection at MSUK.

Co-author / committee member of national guidelines cited in this paper from RCOG \& NICE; co-chair of RCOG abortion taskforce and British Society of Abortion Care Providers (BSACP); primary employment includes work as an abortion care provider as a consultant gynaecologist and medical director. 
Jennifer Starling - Data analysis including statistical advice, writing first draft and subsequent revisions, verification of data.

Nothing to disclose

\section{Copyright/license for publication}

The Corresponding Author has the right to grant on behalf of all authors and does grant on behalf of all authors, a worldwide licence to the Publishers and its licensees in perpetuity, in all forms, formats and media (whether known now or created in the future), to i) publish, reproduce, distribute, display and store the Contribution, ii) translate the Contribution into other languages, create adaptations, reprints, include within collections and create summaries, extracts and/or, abstracts of the Contribution, iii) create any other derivative work(s) based on the Contribution, iv) to exploit all subsidiary rights in the Contribution, v) the inclusion of electronic links from the Contribution to third party material where-ever it may be located; and, vi) licence any third party to do any or all of the above

\section{Data Sharing Statement}

The collated datasets, which include participant data with anonymised identifiers, are held by AA at the University of Texas. Consideration will be given to sharing this with bone fide researchers on application. The original data resides with the co-authors' own institutions. Although the data is de-identified, some relate to very rare events and could therefore result in identification. Therefore data on complications, and data arising from clinical incident reports, will be subject to the same access restrictions as those of the organisation supplying it.

\section{Transparency Statement}

The authors affirm that the manuscript is an honest, accurate, and transparent account of the study being reported; that no important aspects of the study have been omitted; and that any discrepancies from the study as originally planned (and, if relevant, registered) have been explained.

\section{Funding Source}

None

\section{Ethics Statement}

The study was reviewed by the Institutional Review Board (IRB) at the University of Texas at Austin and a determination was made that the research did not meet the criteria for human subjects research as defined in the Common Rule (45 CFR 46) or FDA Regulations (21 CFR 56). Each provider ensured compliance with their own internal ethics and governance systems. The principles of the STROBE statement, and the MARE supplement, were followed.(14)

\section{References}

1. Bearak J, Popinchalk A, Ganatra B, Moller A-B, Tunçalp Ö, Beavin C, et al. Unintended pregnancy and abortion by income, region, and the legal status of abortion: estimates from a comprehensive model for 1990-2019. The Lancet Global Health. 2020.

2. Sedgh G, Bearak J, Singh S, Bankole A, Popinchalk A, Ganatra B, et al. Abortion incidence between 1990 and 2014: global, regional, and subregional levels and trends. Lancet. 2016;388(10041):258-67.

3. Regan L, Glasier A. The British 1967 Abortion Act-still fit for purpose? Lancet. 2017;390(10106):1936-7.

4. National Institute for Health and Care Excellence (NICE). Abortion care. Evidence Review A - Accessibility and sustainability of abortion services. London; 2019 9/2019.

5. O'Shea LE, Hawkins JE, Lord J, Schmidt-Hansen M, Hasler E, Cameron S, et al. Access to and sustainability of abortion services: a systematic review and meta-analysis for the National Institute of Health and Care Excellence - new clinical guidelines for England. Human Reproduction Update. 2020. 
6. WHO Global Observatory for eHealth. Telemedicine: opportunities and developments in Member States: report on the second global survey on eHealth. Geneva: World Health Organization; 2010.

7. Dorsey ER, Topol EJ. Telemedicine 2020 and the next decade. The Lancet. 2020;395(10227):859.

8. Endler M, Lavelanet A, Cleeve A, Ganatra B, Gomperts R, Gemzell-Danielsson K. Telemedicine for medical abortion: a systematic review. BJOG. 2019;126(9):1094-102.

9. Royal College of Obstetricians and Gynaecologists (RCOG), Royal College of Midwives (RCM), Faculty of Sexual \& Reproductive Healthcare (FSRH), British Society of Abortion Care Providers (BSACP). Coronavirus (COVID-19) infection and abortion care. London, UK: Royal College of Obstetricians and Gynaecologists; $202031 / 07 / 2020$.

10. Department of Health \& Social Care (DHSC). The Abortion Act 1967 - Approval of a Class of Places. London: English Government; 2020.

11. Scottish Government. Abortion - Covid-19 - Approval For Mifepristone to be Taken At Home and Other Contingency Measures. Edinburgh: Scottish Government; 2020.

12. Welsh Government. The Abortion Act 1967 - Approval of a Class of Place for Treatment for the Termination of Pregnancy (Wales) 2020. In: Government W, editor. Cardiff: Welsh Government; 2020.

13. Aiken ARA, Guthrie KA, Schellekens M, Trussell J, Gomperts R. Barriers to accessing abortion services and perspectives on using mifepristone and misoprostol at home in Great Britain. Contraception. 2018;97(2):177-83.

14. Creinin MD, Chen MJ. Medical abortion reporting of efficacy: the MARE guidelines. Contraception. 2016;94(2):97-103.

15. Royal College of Obstetricians and Gynaecologists (RCOG). Decision aid for early medical abortion without ultrasound. London: Royal College of Obstetricians and Gynaecologists (RCOG), ; 2020.

16. National Institute for Health and Care Excellence (NICE). Abortion Care. London; 2019 9/25/2019. Report No.: NG140.

17. Royal College of Obstetricians and Gynaecologists (RCOG), Faculty of Sexual \& Reproductive Healthcare (FSRH), British Society of Abortion Care Providers (BSACP). Clinical Guidelines for Early Medical Abortion at Home - England. London; 2019 1/3/2019.

18. Erlank CP, Lord J, Church K. Early medical abortion using telemedicine - acceptability to patients [preprint]. MedRxiv. 2020:2020.11.11.20229377.

19. Meurice M, Whitehouse K, Blaylock R, Chang J, P L. Client satisfaction and experience of home use of mifepristone and misoprostol for medical abortion up to 10 weeks' gestation at British Pregnancy Advisory Service: a cross-sectional evaluation [preprint]. . Authorea. 2020.

20. Ge M, Durham LK, Meyer RD, Xie W, Thomas N. Covariate-Adjusted Difference in Proportions from Clinical Trials Using Logistic Regression and Weighted Risk Differences. Drug information journal : DIJ / Drug Information Association. 2011;45(4):481-93.

21. Department of Health \& Social Care (DHSC). Abortion statistics during COVID-19: data tables. In: Department of Health \& Social Care (DHSC), editor. London: UK Government; 2020.

22. Aiken ARA, Starling JE, Gomperts R, Scott JG, Aiken C. Demand for Self-Managed Online Telemedicine Abortion in Eight European Countries During the COVID-19 Pandemic: A Regression Discontinuity Analysis [preprint]. MedRxiv. 2020:2020.09.15.20195222.

23. Upadhyay UD, Desai S, Zlidar V, Weitz TA, Grossman D, Anderson P, et al. Incidence of emergency department visits and complications after abortion. Obstet Gynecol. 2015;125(1):175-83. 
24. Centers for Disease Control and Prevention (CDC). Ectopic pregnancy-United States, 1990-1992. MMWR Morb Mortal Wkly Rep. 1995;44(46-8):46-8.

25. National Institute for Health and Care Excellence (NICE). Ectopic pregnancy and miscarriage: diagnosis and initial management. London; 2019 4/17/2019. Report No.: NG126.

26. Shannon C, Brothers LP, Philip NM, Winikoff B. Ectopic pregnancy and medical abortion. Obstet Gynecol. 2004;104:161-7.

27. Mol BW, van der Veen F, Bossuyt PM. Symptom-free women at increased risk of ectopic pregnancy: should we screen? Acta Obstet Gynecol Scand. 2002;81(7):661-72.

28. Clark WH, Gold M, Grossman D, Winikoff B. Can mifepristone medical abortion be simplified? A review of the evidence and questions for future research. Contraception. 2007;75(4):245-50.

29. Bracken H, Clark W, Lichtenberg ES, Schweikert SM, Tanenhaus J, Barajas A, et al. Alternatives to routine ultrasound for eligibility assessment prior to early termination of pregnancy with mifepristonemisoprostol. BJOG. 2011;118(1):17-23.

30. Raymond EG, Grossman D, Mark A, Upadhyay UD, Dean G, Creinin MD, et al. Commentary: No-test medication abortion: A sample protocol for increasing access during a pandemic and beyond. Contraception. 2020 .

31. Harris LH, Grossman D. Complications of Unsafe and Self-Managed Abortion. N Engl J Med. 2020;382(11):1029-40.

Tables and Figures

Table 1: Clinical and demographic characteristics in the traditional and telemedicine-hybrid cohorts $(\mathrm{n}=52,142)$

\begin{tabular}{llll}
\hline Patient Characteristic & $\begin{array}{l}\text { Traditional } \\
(\mathbf{n = 2 2 , 1 5 8 )}\end{array}$ & $\begin{array}{l}\text { Telemedicine-hybrid } \\
\mathbf{( n = 2 9 , 9 8 4 )}\end{array}$ & P-value \\
\hline $\begin{array}{l}\text { Mean gestational } \\
\text { age in weeks (s.d.) }\end{array}$ & $6.4(1.3)$ & $6.0(1.4)$ & $<0.001$ \\
$\begin{array}{l}\text { Gestational age at } \\
\text { treatment }\end{array}$ & & \\
6 weeks and under & $5,582(25.2)$ & $11,947(39.8)$ & \\
Over 6 weeks & $16,576(74.8)$ & $18,037(60.2)$ & $<0.001$ \\
Mean age in years & $27.8(6.6)$ & $28.5(6.7)$ & $<0.001$ \\
(s.d.) & & & \\
Ethnicity & $2,038(9.2)$ & $2,652(8.8)$ & \\
Asian & $1,656(7.5)$ & $2,282(7.6)$ & \\
Black & $1,004(4.5)$ & $1,361(4.5)$ & \\
Multiracial & $15,840(71.5)$ & $20,910(69.7)$ & \\
White & $489(2.2)$ & $638(2.1)$ & \\
Other & $1,131(5.1)$ & $2,141(7.1)$ & \\
Unknown & & \\
Previous abortions & $13,098(59.1)$ & $16,741(55.8)$ & $<0.001$ \\
0 & $9,060(40.9)$ & $13,243(44.2)$ & \\
1+ & & & \\
Parity & $10,133(45.7)$ & $11,741(39.2)$ & $<0.001$ \\
0 & $12,025(54.3)$ & $18,243(60.8)$ & \\
$1+$ & &
\end{tabular}




\begin{tabular}{llll}
\hline Patient Characteristic & $\begin{array}{l}\text { Traditional } \\
(\mathbf{n = 2 2 , 1 5 8 )}\end{array}$ & $\begin{array}{l}\text { Telemedicine-hybrid } \\
(\mathbf{n = 2 9 , 9 8 4 )}\end{array}$ & P-value \\
\hline $\begin{array}{l}\text { Mean waiting time } \\
\text { in days (s.d.) }\end{array}$ & $10.7(19.9)$ & $6.5(13.5)$ & $<0.001$ \\
\hline
\end{tabular}

Table 2: Comparison of effectiveness of medical abortions conducted in the traditional and telemedicine-hybrid cohorts $(n=52,142)$

\begin{tabular}{llll}
\hline Outcome & Traditional $\mathbf{n = 2 2 , 1 5 8}$ & $\begin{array}{l}\text { Telemedicine-hybrid } \\
\mathbf{n = 2 9 , 9 8 4}\end{array}$ & P-value \\
\hline $\begin{array}{l}\text { Successful medical } \\
\text { abortion }\end{array}$ & $\mathbf{2 1 , 7 6 9 ( 9 8 . 2 )}$ & $\mathbf{2 9 , 6 1 8 ( 9 8 . 8 )}$ & $\mathbf{1 . 0}$ \\
$\begin{array}{l}\text { Unsuccessful } \\
\text { medical abortion }\end{array}$ & $\mathbf{3 8 9 ( 1 . 8 )}$ & $\mathbf{3 6 6}(\mathbf{1 . 2})$ & \\
$\begin{array}{l}\text { Continuing pregnancy } \\
\text { treated with surgical } \\
\text { management }\end{array}$ & $161(0.7)$ & $150(0.5)$ & 0.268 \\
$\begin{array}{l}\text { Continuing pregnancy } \\
\text { opted to continue or } \\
\text { unknown outcome }\end{array}$ & $3(0.01)$ & $8(0.03)$ & \\
$\begin{array}{l}\text { Retained products } \\
\text { treated with surgical } \\
\text { management (ERPC) }\end{array}$ & $225(1.0)$ & $208(0.7)$ & \\
\hline
\end{tabular}

Note: As explained in the methods section, the p-value for successful medical abortion is the co-variate adjusted p-value (i.e. all differences in patient clinical and demographic characteristics, including gestational age, are controlled for) and was calculated using a hypothesis test where the null hypothesis is that the traditional cohort has the same effectiveness rate as the telemedicine-hybrid cohort and the alternative hypothesis is that the traditional cohort has a higher effectiveness rate than the telemedicine-hybrid cohort. The p-value for unsuccessful medication abortion is for the chi-squared test of whether the distribution of types of failure differ between the cohorts.

Table 3: Comparison of significant adverse events following medical abortions conducted in the traditional and telemedicine-hybrid cohorts $(n=52,142)$

\begin{tabular}{llll}
\hline Outcome & $\begin{array}{l}\text { Traditional } \\
(\mathbf{n = 2 2 , 1 5 8 )}\end{array}$ & $\begin{array}{l}\text { Telemedicine-hybrid } \\
(\mathbf{n = 2 9 , 9 8 4 )}\end{array}$ & P-value \\
\hline $\begin{array}{l}\text { Haemorrhage } \\
\text { requiring } \\
\text { transfusion }\end{array}$ & $8(0.04)$ & $7(0.02)$ & 0.557 \\
$\begin{array}{l}\text { Infection requiring } \\
\text { hospital admission }\end{array}$ & $0(0.0)$ & $0(0.0)$ & \\
$\begin{array}{l}\text { Major surgery } \\
\text { Death }\end{array}$ & $0(0.0)$ & $0(0.0)$ & \\
\hline
\end{tabular}

Note: As explained in the methods section, the p-value was calculated using a hypothesis test where the null hypothesis is that the traditional cohort the same rate of adverse events than the telemedicine-hybrid 
cohort and the alternative hypothesis is that the traditional cohort has a lower rate of adverse events than the telemedicine-hybrid cohort.

Table 4: Significant outcomes among patients presenting for medical abortion in the traditional and telemedicine-hybrid cohorts $(n=52,218)$

\begin{tabular}{llll}
\hline Outcome & $\begin{array}{l}\text { Traditional } \\
(\mathbf{n = 2 2 , 1 9 7 )}\end{array}$ & $\begin{array}{l}\text { Telemedicine-hybrid } \\
(\mathbf{n = 3 0 , 0 2 1})\end{array}$ & P-value \\
\hline $\begin{array}{l}\text { Ectopic Managed } \\
\begin{array}{l}\text { Pre-Treatment } \\
\text { Ectopic Managed }\end{array}\end{array}$ & $37(0.17)$ & $39(0.13)$ & 0.796 \\
$\begin{array}{l}\text { Post-Treatment } \\
\text { Gestational age }\end{array}$ & $0(0.01)$ & $10(0.03)$ & 0.123 \\
later than expected* & & $11(0.04)$ & $\mathrm{N} / \mathrm{A}$ \\
\hline
\end{tabular}

Note: The column Ns include patients who presented for an EMA but did not receive one because their ectopic pregnancy was identified pre-treatment.

*Note: The column Ns for the gestational age later than expected category are the same as those in Table 3 , (all EMAs performed in the two cohorts, i.e. $\mathrm{N}=52,142$ ).

Figure 1 - Summary of early medical abortion care management during COVID-19 pandemic (adapted from RCOG Coronavirus (COVID-19) infection and abortion care - information for healthcare professionals) (9)

[Note for editors - permission for reproduction obtained from RCOG]

Image from page 4: 2020-07-31-coronavirus-covid-19-infection-and-abortion-care.pdf (rcog.org.uk)

If preferred, can provide original files in original Word format 


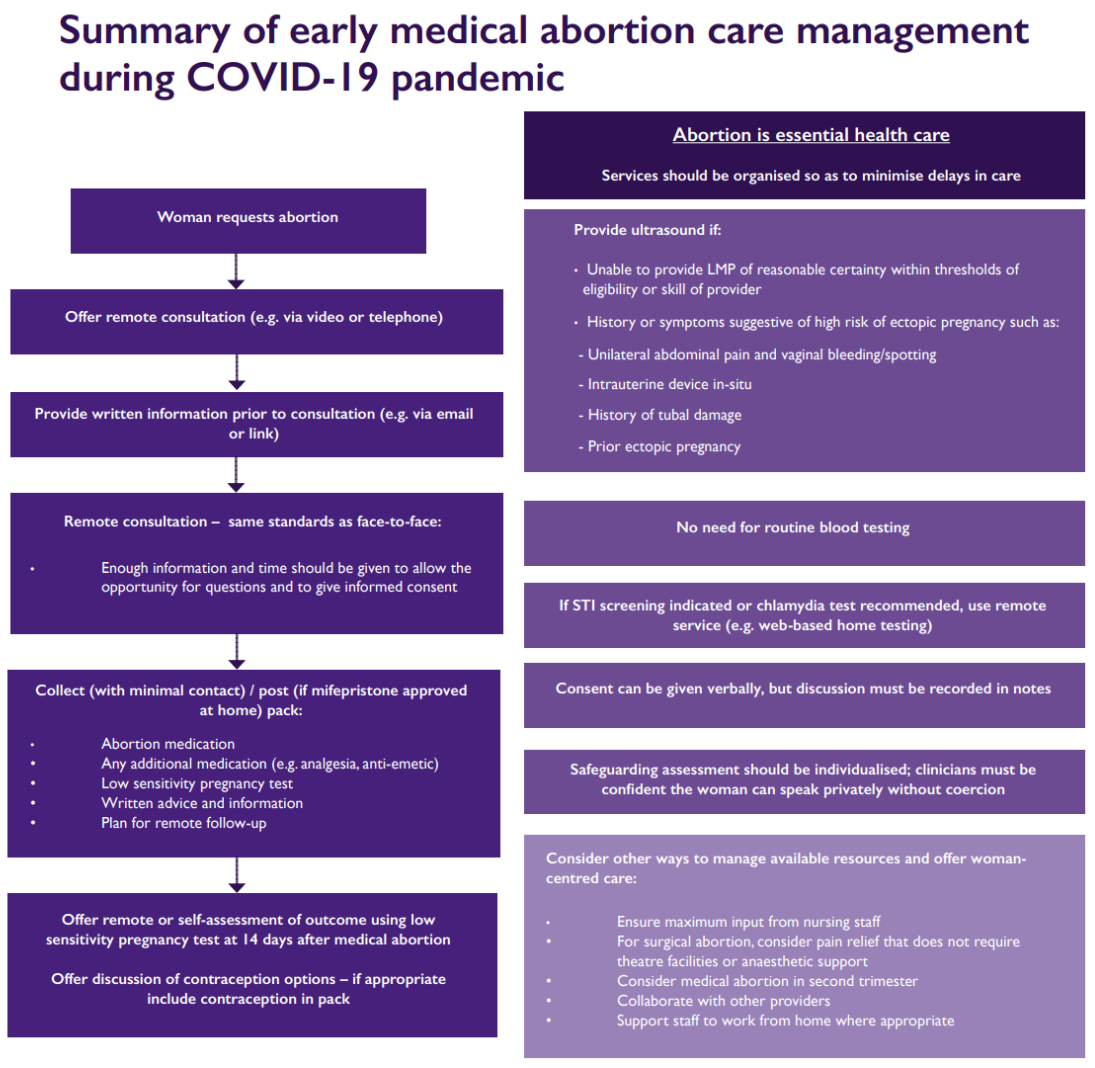

Figure 2 - Decision aid for early medical abortion without ultrasound (adapted from RCOG Coronavirus (COVID-19) infection and abortion care - information for healthcare professionals) (15)

[Note for editors - permission for reproduction obtained from RCOG]

Image from: 2020-06-04-decision-aid-for-early-medical-abortion-without-ultrasound.pdf (rcog.org.uk)

If preferred, can provide original files in original Powerpoint format 

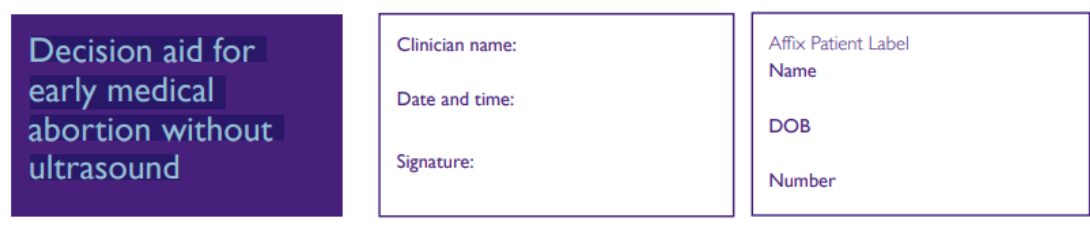

Questions to ask the patient
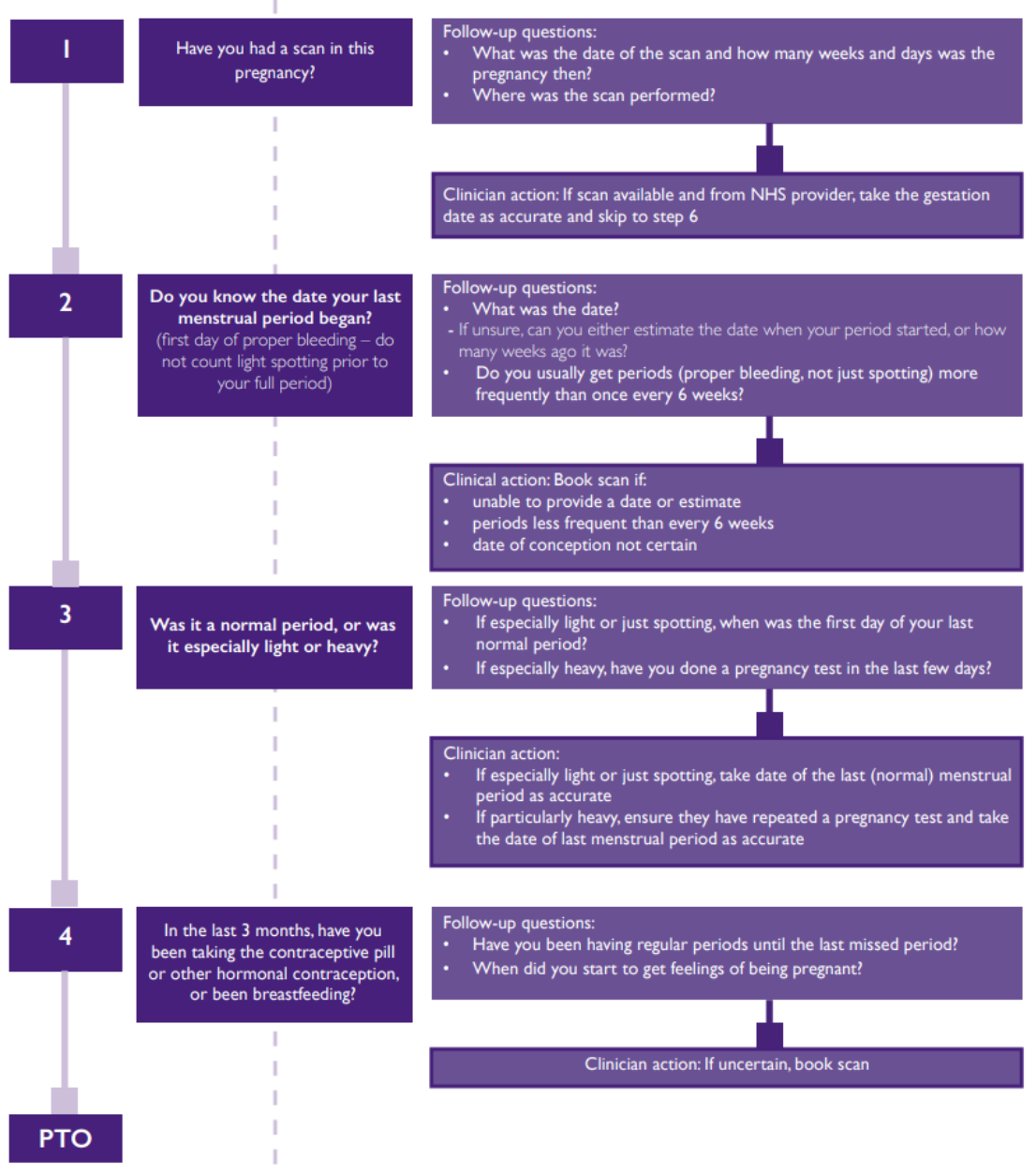

Follow-up questions:

- Have you been having regular periods until the last missed period?

When did you start to get feelings of being pregnant? 


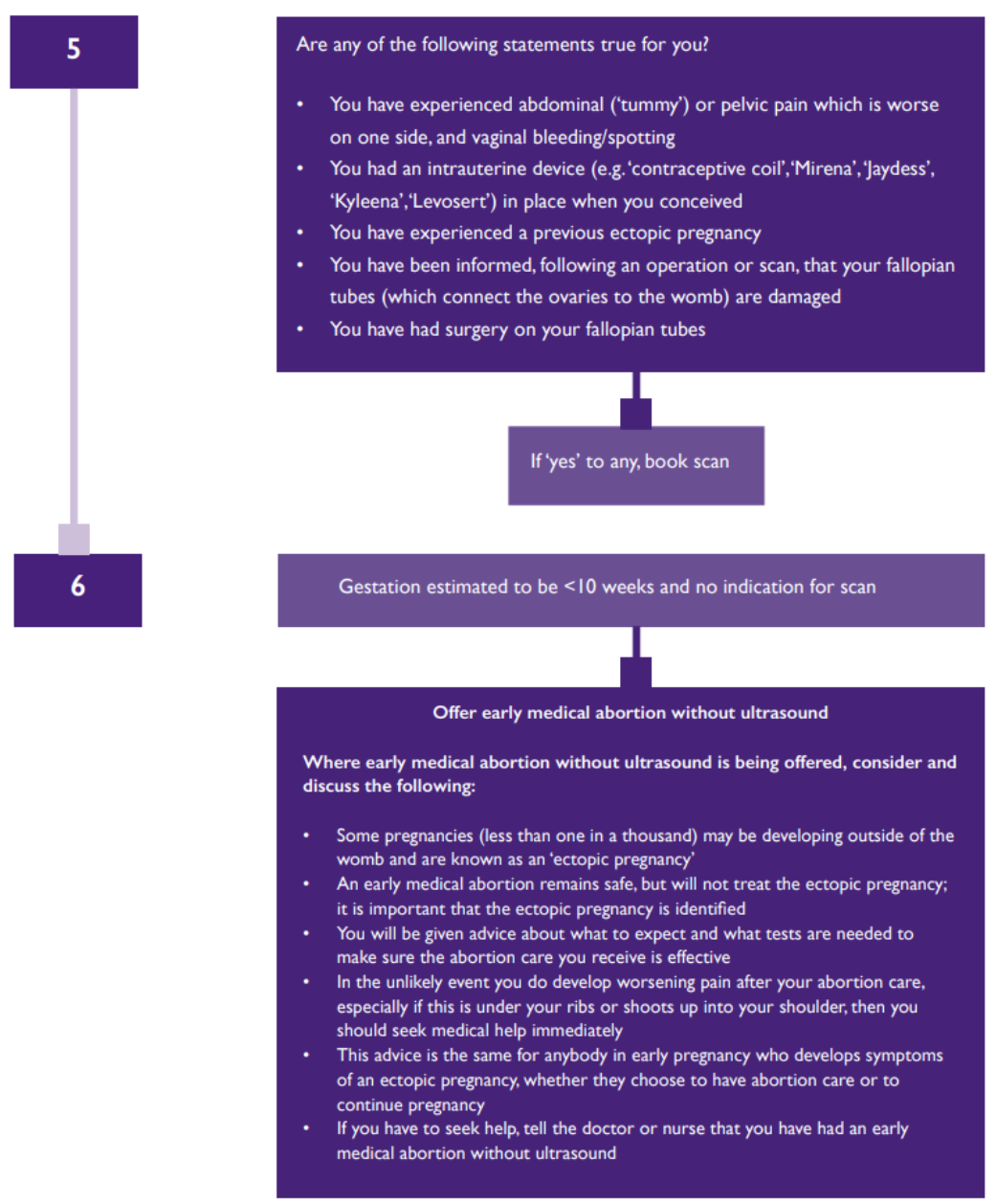

* Royal College of Obstetricians \& Gynaecologists

Royal College of Obstetricians and Gynaecologists, 10-18 Union Street, London, SEI ISZ T: +44 (0)2077726200 E: covid-19@rcog.org.uk W: rcog.org.uk Registered Charity No. 213280 